\title{
A method of predicting the time of failure of a rolling bearing
}

\author{
Dmitry Liakhmanov ${ }^{1}$, Vasily Gay ${ }^{1}$, and Igor Polyakov ${ }^{1, *}$ \\ ${ }^{1}$ NNSTU named after R. E Alekseev, Nizhny Novgorod, Russia
}

\begin{abstract}
This article considers the problem of predicting wear and breakage of machine components using rolling bearings. Any mechanism wears out over time and, accordingly, the risk of breakage increases. This creates the need for timely maintenance of worn out parts of machinery. The solution of the problem consists in the development of new models and methods for predicting the condition of bearings in the nodes and mechanisms. The developed methods, unlike known ones, should allow us to increase the accuracy of the residual resource prediction, working in the absence of accumulated statistics on faults. To solve the problem, it is proposed to use the theory of active perception, which allows solving problems associated with the use of known methods of pattern recognition.
\end{abstract}

\section{Introduction}

Mechanisms wear out over time and, accordingly, the risk of their breakage increases. This creates the need for timely maintenance of worn out parts of machinery. One of the most important elements of almost any mechanism is the rolling bearing. Therefore, the bearing can be considered as a critical node, since its failure reduces the safety and operability of the machine. There are various strategies for planning maintenance and repair of technical equipment. The most effective strategy is the maintenance strategy for the equipment. This strategy allows you to optimize the maintenance by predicting the occurrence of breakages [1-3].

Methods for predicting the residual life of the device can be divided into three groups: based on the physical model of the device, based on monitoring data devices and hybrid [1].

Forecasting methods based on the physical model are usually represented as a set of algebraic or differential equations and describe the behavior of the physical system under investigation, including its destruction. The advantage of this approach is that it provides accurate results; their disadvantage is the complexity of creating a model due to the nonlinearity of the processes occurring in the devices.

Data-based prediction methods perform conversion of device monitoring data to the information used for future prediction. The model of destruction of the device is created only on the basis of data provided by the monitoring system without using the analytical model of the system or its physical parameters. The forecast is formed on the basis of

\footnotetext{
${ }^{*}$ Corresponding author: polyakovigor92@gmail.com
} 
artificial intelligence methods or statistical methods. Estimation of the residual resource based on the data is performed in two stages: creating a model of the device's behavior in time and using this model to predict the residual resource of the device.

The hybrid prediction method combines approaches based on the physical model and data-based.

At present, the methods of forecasting based on monitoring data are most actively developed. For example, there are competitions devoted to the development of these prediction methods.

The main difficulties in the development of data-based prediction methods are associated with the presence of distortions in the readings of sensors and with the complexity of selecting characteristics possessing the property of monotony and the separation of the trend. These properties are necessary to ensure an accurate estimate of the remaining life of the device.

In this connection, the scientific problem related to the development of models and methods for estimating the residual life of the rolling bearing is of current interest.

The scientific significance of solving the problem consists in the development of new models and methods for predicting the state of bearings in nodes and mechanisms. The developed methods, in contrast to the known ones, should allow increasing the accuracy of the prediction of the residual resource, working in conditions of a priori uncertainty (the absence of accumulated statistics on faults).

Currently, when creating systems for servicing mechanisms, the use of data mining is a acute. With regard to the task, this will improve their reliability and safety.

The scientific novelty of the task is to use for the solution of the problem of vibrodiagnostics and forecasting the theory of active perception [4], which allows solving problems associated with the use of known methods of pattern recognition.

The task for which this work is directed is the development of models and methods for predicting the residual life of a rolling bearing based on data.

Within the framework of the task, it is planned to develop models for estimating the residual life of a rolling bearing based on the proposed systems of characteristics. It is also necessary to carry out experimental studies of the developed models and methods of forming systems of characteristics, in order to assess the accuracy of the forecast of the remaining resource.

Let's consider the well-known work in the field of the problem posed. The paper [5] is considered in which the vibration signal analysis is carried out using the principal component method used to reduce the dimension of the original vibration features by removing redundant features. This method allows you to reduce the final amount of calculations, but this method does not realize the full potential of the available data. To calculate the remaining running time of the bearing, a method of data description using support vectors is used, based on the principles of minimizing structural risks. It is used to relate training data to the hypersphere region, so the boundary of the resulting radius of the hypersphere can be used as an indicator of the bearing state. This method shows good results in this kind of calculations and it was also decided to use it in one of the stages. The paper [6] is considered in which the vibration signal analysis is carried out using the Bayesian Monte Carlo simulation method, and the calculation of the remaining operation time of the bearing is realized using the moving average spectral excess method and the regression method of support vectors. Experiments in both studies were carried out on the same data set used in this work [7]. The results have prediction accuracy up to $85-95 \%$, however in some cases there is a significant drop in accuracy. The methods used in the articles are quite expensive in terms of computational complexity. In analyzing these 
studies, it was concluded that there is an opportunity to improve the accuracy of work and reduce the computational complexity, using the proposed methods

\section{Information prediction model}

The information model consists of the part responsible for analyzing the vibration signal and part of the calculation of the remaining operation time of the bearing. Introducing the vibration analysis system as an image recognition system, we distinguish three stages of data processing: preliminary processing, calculation of features and decision making. The structure is shown in Figure 1.

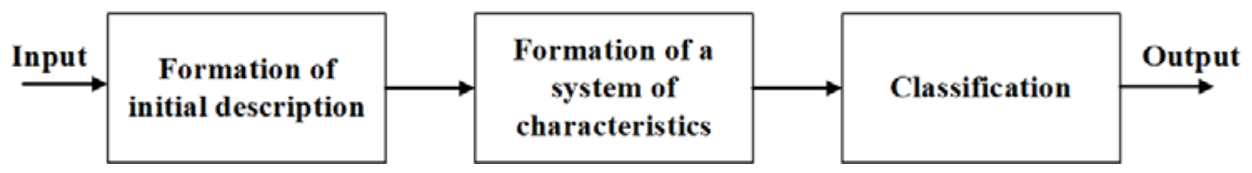

Fig. 1. Structure of the recognition system.

The first and second stages are proposed to be implemented using the theory of active perception, which makes it possible to reduce computational complexity, in comparison with similar methods. Preliminary processing consists in performing a Q-transformation, which involves applying addition operations to the segments of the original signal. The formation of an indicative description of the original signal consists in applying to the signal $g$ the set of Walsh filters of the Harmut system [8]. Sequential application to the signal of the Q-transform and the filter system implements the U-transformation, which is basic in the theory of active perception. The U-transformation has the minimum possible computational complexity, because its implementation uses the simplest operations addition and subtraction. Standard transformations require the implementation of convolution, and at the level of weighting coefficients - the operations of arithmetic multiplication. The theory of active perception is not limited only to the formation of the spectral representation of the signal. The theory includes the section "Algebra of groups", devoted to the analysis of the dependencies between the spectral coefficients of the expansion. The observed dependencies allow their use at the decision-making and understanding stages of the analyzed signal.

The classification stage is proposed to be realized using the One-class SVM machine learning method, which works well for finding deviations in problems with a lot of standard data. Accumulation of variances is recorded and when a certain value is exceeded, it displays the boundary of the beginning of the so-called zones of anomalous data. This makes it possible to reduce the number of measurements used at the forecasting stage, taking into account only the data from the anomalous zone.

Further, the cumulative mean square deviation is calculated from the anomalous data array obtained in the previous step. The obtained data approximates the final part of the curve using a parabola, the beginning of the approximation is calculated using the basis function [4]. One of the coefficients of the resulting parabola, as a result, signals the state of the bearing (its wear rate over the measured time interval). Based on this, the remaining running time of the bearing is calculated (figure 2). 


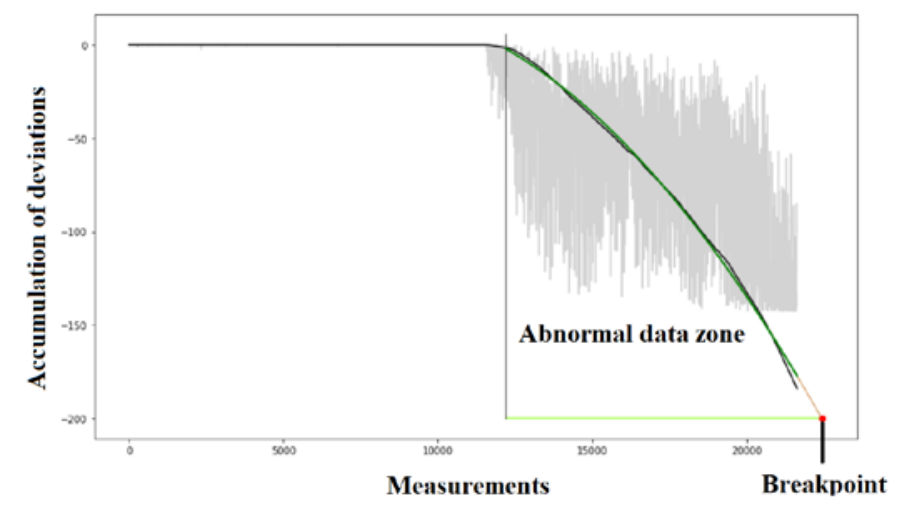

Fig. 2. Calculation of the remaining running time of the bearing.

\section{Computational experiment}

The experiment was carried out on a data packet presented in [7] (Figure 3). Each set is the result of testing six bearings before failure. Each set consists of files containing vibration signals (horizontal and vertical acceleration):

1) Sampling frequency: $25.6 \mathrm{kHz}$;

2) Records: 2560 measurements (1/10 s long) recorded with an interval of 10 seconds.

For each data set corresponding to the test results of one of the bearings, the total time of data recording from the bearing, up to the moment of failure, is indicated.

The experiment was carried out with data from three pairs of bearings operating under different conditions:

1) $1800 \mathrm{rpm}$ and applied radial load of $4000 \mathrm{~N}$;

2) $1650 \mathrm{rpm}$ and the applied radial load of $4200 \mathrm{~N}$;

3) $1500 \mathrm{rpm}$ and the applied radial load of $5000 \mathrm{~N}$.

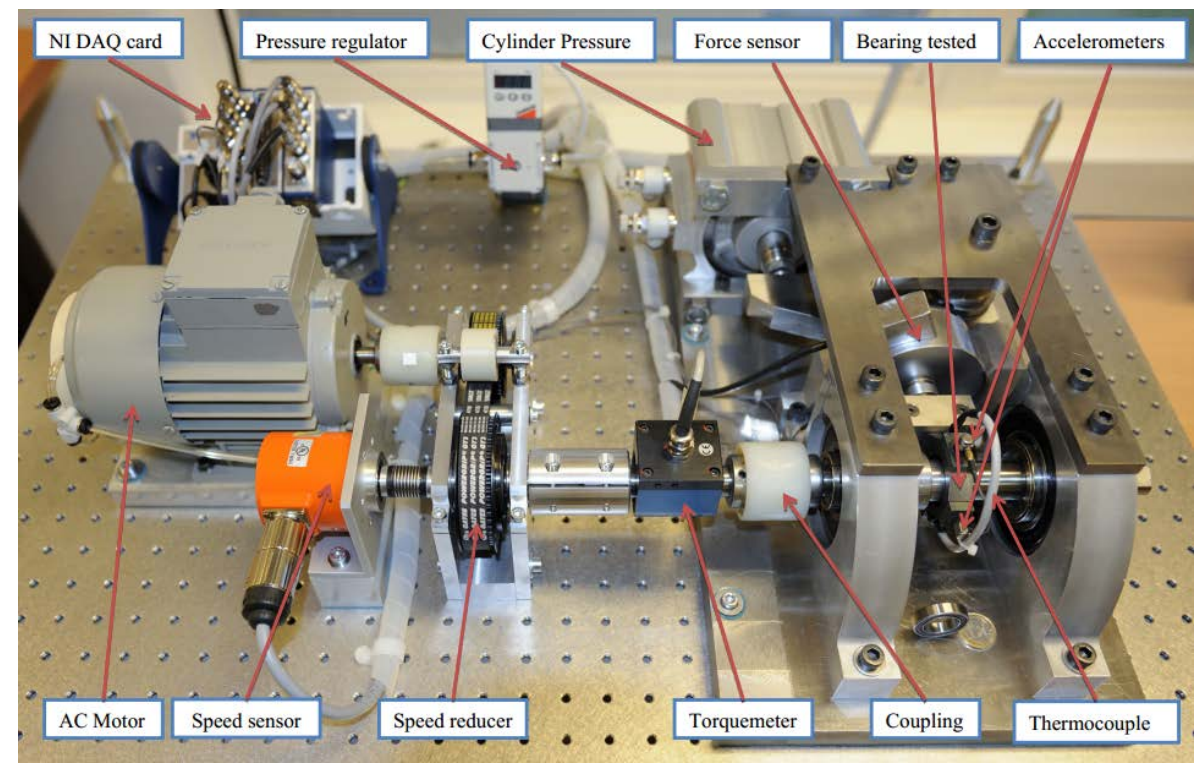

Fig. 3. Installation for testing bearings. 
To test the efficiency of the proposed method, a series of experiments was carried out. The computer that makes the calculations has an Intel Core i7 $3.6 \mathrm{GHz}$ processor and $8 \mathrm{~GB}$ of RAM. The stages of the signal preprocessing and calculation of the characteristics are performed in the $\mathrm{R}$ language, the decision-making and residual resource calculations are performed in Python. The program works in two steps: At the first step the system provides data for training, including vibration data, corresponding to the operation of the bearing in good condition. In the second step, the system conducts a study of all available information on the bearing condition and produces a result that includes the remaining operating time before a critical failure.

Training the program was carried out on $30 \%$ of the data, a prediction model was constructed and compared with actual indications. A greater number of results showed a prediction of accuracy with the methods considered above [6], but there were cases where the accuracy fell significantly, which indicates the need for further work on the algorithm (Table 1).

Table 1. Errors in remaining useful life estimation.

\begin{tabular}{|l|l|l|l|l|l|l|l|l|l|l|l|}
\hline & \multicolumn{1}{|l|}{ Obtained results } \\
\hline Bearing & $1 \_3$ & $1 \_4$ & $1 \_5$ & $1 \_6$ & $1 \_7$ & $2 \_3$ & $2 \_4$ & $2 \_5$ & $2 \_6$ & $2 \_7$ & $3 \_3$ \\
\hline Error (\%) & 0,35 & 44,2 & -95 & -428 & $-22,6$ & -244 & -37 & 28,4 & -46 & -259 & 60 \\
\hline & Results from [6] \\
\hline Bearing & $1 \_3$ & $1 \_4$ & $1 \_5$ & $1 \_6$ & $1 \_7$ & $2 \_3$ & $2 \_4$ & $2 \_5$ & $2 \_6$ & $2 \_7$ & $3 \_3$ \\
\hline Error (\%) & 37 & 80 & 9 & -5 & -2 & 64 & 10 & -440 & 49 & -317 & 90 \\
\hline
\end{tabular}

\section{Conclusion}

We carried out scientific research and experimental works in the field of creation of forecasting systems. As a result, the results of the work on the topic under consideration can include the development of a data-based approach. The solution of the problem was carried out from the position of the theory of active perception. The results of computational experiments confirm the effectiveness of the proposed methods. Increase the accuracy of the work can be done with the help of a more accurate algorithm setting. The created system has the potential for further development and can be used for educational purposes. In addition, a study of existing forecasting systems was carried out, their effectiveness in solving the problem of calculating the forecast of the bearing state was indicated.

\section{References}

1. 1. E.I. Gromakov, T.V. Alexandrova, A.V. Rudachenko, A. M. Malyshenko Maintenance and repair of the equipment condition using Shewhart charts, News of the Tomsk Polytechnic University. V. 317. № 5, P. 112-117. (2010)

2. A. Heng, A. C. Tan, J. Mathew, N. Montgomery, D. Banjevic, and A. K. Jardine, "Intelligent condition-based prediction of machinery reliability," Mechanical Systems and Signal Processing, vol. 23, no. 5, pp. 1600 - 1614, (2009) 
3. A. K. Jardine, D. Lin, and D. Banjevic, "A review on machinery diagnostics and prognostics implementing condition-based maintenance," Mechanical Systems and Signal Processing, vol. 20, no. 7, pp. $1483-1510$ (2006)

4. 4. Utrobin V.A .Information models of the visual perception system for computer image processing problems. - Nizhny Novgorod: NNSTU. n.a R.E. Alekseev, 2001.- P. 234. (2001)

5. T. Benkedjouh, K. Medjaher, N. Zerhouni, S. Rechak. Fault prognostic of bearings by using support vector data description, 2012 IEEE Conference on Prognostics and Health Management (2012)

6. E. Sutrisno; H. Oh; A. Sai Sarathi Vasan; M. Pecht. Estimation of remaining useful life of ball bearings using data driven methodologies, 2012 IEEE Conference on Prognostics and Health Management (PHM) (2012)

7. http://www.femto-st.fr/en/Research-departments/AS2M/Research-groups/PHM/IEEEPHM-2012-Data-challenge.php

8. V. A. Utrobin, Physical interpretations of elements of image algebra, Uspekhi Fizicheskikh Nauk. - T. 174, No. 10. - P. 1089-1104. (2004) 